\title{
Vortex-to-Polarization Phase Transformation Path in $\mathrm{Pb}(\mathrm{ZrTi}) \mathrm{O}_{3}$ Nanoparticles
}

\author{
Ivan Naumov and Huaxiang Fu \\ Department of Physics, University of Arkansas, Fayetteville, AR 72701, USA
}

(Dated: May 31, 2022)

\begin{abstract}
Phase transformation in finite-size ferroelectrics is of fundamental relevance for understanding collective behaviors and balance of competing interactions in low-dimensional systems. We report a first-principles effective Hamiltonian study of vortex-to-polarization transformation in $\mathrm{Pb}\left(\mathrm{Zr}_{0.5} \mathrm{Ti}_{0.5}\right) \mathrm{O}_{3}$ nanoparticles, caused by homogeneous electric fields normal to the vortex plane. The transformation is shown to (1) follow an unusual macroscopic path that is symmetry nonconforming and characterized by the occurrence of a previously unknown structure as the bridging phase; (2) lead to the discovery of a striking collective phenomenon, revealing how ferroelectric vortex is annihilated microscopically. Interactions underlying these behaviors are discussed.

PACS numbers: 77.80.Bh, 77.22.Ej, 64.70.Nd, 77.84.Dy
\end{abstract}


Vortex is a circularly ordered structure phase of broad interest, which has been found in ferromagnets (FM) [1], superconductors [2], and Bose-Einstein condensated atoms [3]. Recently, the vortex phase formed by electric dipoles, as hinted by Ginzburg et al. when exploring the possibility of superdiamagnetism [4], was revealed to exist in ferroelectric (FE) nanoparticles. [5] It was shown [5] that the macroscopic toroid moment undergoes a spontaneous transition from being null at high temperature to nonzero at low temperature, acting as the order parameter of the vortex phase. In FEs, another ordered structure of general concern is the ferroelectric phase with an out-of-plane polarization[6]. Transformation between these two ordered structures in FE nanoparticles, by electric fields perpendicular to the vortex plane, is a phase transition of fundamental importance, since (i) Phase transformation in finite-size systems has been long known as a fundamental subject [7]; (ii) The vortex-to-polarization transformation involves two phases of completely different order parameters (namely, toroid moment in vortex phase versus polarization in ferroelectric phase), and is a critical example to study collective mechanism in terms of how order parameter nucleates and/or disappears within a single domain; (iii) Participation of external electric fields during transformation alters and re-organizes the delicate balance among competing interactions inside FEs [8], and may lead to new structural phases that are not possible in the zero-field case; (iv) It concerns the interaction between FE vortex and electric fields. Vortex, as a whole, does not interact with electric fields because of vanished net polarization; but individual dipoles do, which explains why collective behavior during the vortex transformation is interesting.

Technologically, the FE vortex phase and the polarization phase both are of considerable significance. [9, 10] The former promises to increase the storage density of nonvolatile ferroelectric random access memories by five orders of magnitude[5], while FEs in polarization phase exhibit large strain and electromechanical response, which is of widespread use in piezoelectric transducers and actuators. [10, 11] Nowadays, FE particles[12], wires [13], and nanotubes [14] were all made possible in experiments.

Despite the fact that vortices exist both in FE and FM particles, they are different in key aspects arising from the profound difference between electrostatic and magnetostatic interactions. First, the demagnetizing energy in FM particles is $10^{3}$ times smaller than the depolarizing counterpart in FE particles of the same size, leading to the fact that for particles less than 10nm the dominant interaction in FMs is the short-range (SR) exchange 
interaction which favors magnetization, much like in bulk 15, 16]. As size increases, the demagnetization energy (scaling approximately as $\mathrm{N}^{\frac{5}{3}}$ where $\mathrm{N}$ is the number of dipoles in particle) becomes more important than the SR exchange energy (scaling as N), thereby vortex turns favorable until domains start to play a role. Indeed, the minimum threshold size for magnetic $\mathrm{Ni}_{80} \mathrm{Fe}_{14} \mathrm{Mo}_{5}$ particles to adopt a vortex structure is experimentally found as $100 \mathrm{~nm}$ in diameter (particles less than this size exhibit instead magnetization). [17] This is in sharp contrast to the situation in FE nanoparticles where the depolarizing energy is dominant and which exhibit a vortex phase as small as $4 \mathrm{~nm}$. [5] The balance between LR and SR interactions is thus profoundly different in two materials. Furthermore, because of the large depolarizing energy, the shape and anisotropy are insignificant for FE particles when size is small $(<10 \mathrm{~nm})$. In fact, FE vortices have been found in particles of cylindrical[5], cubic[18], and rectangular shapes. As a result of these differences, the knowledge of transforming magnetic vortex is not suitable for FE nanoparticles.

Here we perform ab-initio based simulations on vortex-to-polarization phase transformation, induced by homogeneous electric fields normal to the vortex plane, in nanoparticles made of technologically important $\mathrm{Pb}\left(\mathrm{Zr}_{0.5} \mathrm{Ti}_{0.5}\right) \mathrm{O}_{3}(\mathrm{PZT})$ solid solution. These studies lead to (1) the discovery of a critical new structure that bridges the transformation between two phases of unlike order parameters, (2) an unusual collective mechanism showing how FE vortex is annihilated, (3) the existence of a previously unknown hysteresis, and (4) an innovative approach for reading FE toroid moment. Neither the phase transformation path reported here, nor the unusual dipole behaviors associated with this path, occur for inplane electric fields (which cause only dipole flipping [18]). Further, inplane fields do not produce out-of-plane polarization that is most utilized in practice.

We use first-principles derived effective Hamiltonian [19, 20] and Monte Carlo (MC) simulations to determine dipole configuration. Calculations are performed for PZT nanostructures of cylindrical shape, with diameter $d$ ranging from 9 to 25 and height $h=14$ (both $d$ and $h$ are in units of bulk pseudo-cubic lattice parameter $a=4.0 \AA$ ). The crystallographic [001] direction is chosen as the cylindrical z-axis, with the [100] and [010] directions being the $x$ and $y$ axes. Initial dipole configuration of vortex is obtained from annealing simulations with temperature decreased in small step. Electric field of varied strength is applied along the $+z$ direction at a fixed temperature of $64 \mathrm{~K}$, and is coupled with dipole $\mathbf{p}_{i}$ at cell $i$ by $-\sum_{i} \mathbf{E} \cdot \mathbf{p}_{i}$ as described in Ref.21. Dipole-dipole interaction in nanoparticles is handled in real 
space.[18] Typically 10000 MC sweeps are used to simulate dipole responses at each field. Outcome of simulation is the soft-mode vector field $\left\{\mathbf{u}_{i}\right\}$ (which is directly proportional to local dipole $\mathbf{p}_{i}$ ), homogeneous strain $\left\{\eta_{i}\right\}$, and inhomogeneous cell shape.

Figure 1 a depicts the collective behaviors of toroid moment, $\mathbf{G}=\frac{1}{2 \mathrm{~N}_{\mathrm{c}}} \sum_{i} \mathbf{r}_{i} \times \mathbf{p}_{i}$, and net polarization, $\mathbf{P}=\frac{1}{\mathrm{~N}_{\mathrm{c}} \Omega} \sum_{i} \mathbf{p}_{i}$ (where $\mathrm{N}_{\mathrm{c}}$ is the number of bulk cells and $\Omega$ is the cell volume), that develop in a $\mathrm{d}=19$ nanodisk as the strength of electric field varies. When electric field is small and below a critical value $\mathrm{E}_{c, 1}=1.5 \mathrm{~V} / \mathrm{nm}$, the disk shows only a $\mathrm{G}_{z}$ toroid component while $\mathrm{G}_{x}$ and $\mathrm{G}_{y}$ are null. The system in the $\mathrm{E} \leq \mathrm{E}_{c, 1}$ field region thus retains the same macroscopic toroid symmetry as in zero field (This structure phase will be denoted as phase I hereafter). As the field reaches $\mathrm{E}_{c, 1}$, the $\mathrm{G}_{z}$ moment declines only slightly as compared to the initial zero-field value, and meanwhile, a net polarization of $0.3 \mathrm{C} / \mathrm{m}^{2}$ develops. In fact, this magnitude of polarization is large and comparable to the value found in bulk $\mathrm{BaTiO}_{3} \cdot[10$, 21] The vortex response of phase $\mathrm{I}$ is thus characterized by the coexistence of strong toroid moment and large polarization, both pointing along the cylindrical z-axis. We further numerically find that the toroid moment in phase I responds to the $\mathbf{E}$ field by accurately following a quadratic scaling law as $\mathrm{G}_{\mathrm{z}}(E)=\mathrm{G}_{0}-\sigma \mathrm{E}^{2}$, where $\mathrm{G}_{0}$ is the zero-field $\mathrm{G}_{z}$ moment and the $\sigma$ coefficient is determined to be 285.5 e $\AA^{4} / \mathrm{V}^{2}$ for the $\mathrm{d}=19$ disk.

The system behaves in a markedly different fashion as the electric field exceeds $\mathrm{E}_{c, 1}$, manifested in Fig 1a by the dramatic occurrence of a nonzero $\mathrm{G}_{y}$ component and simultaneously a sharp decline of the $\mathrm{G}_{z}$. Being perpendicular to the initial $\mathrm{G}_{z}$ moment, the appearance of the $\mathrm{G}_{y}$ moment deviates the system from continuing to possess macroscopic cylindrical symmetry, and the resulting symmetry-broken new structure is to be denoted as phase II. Thus for the first time the ferroelectric vortex, under the application of a uniform perpendicular field, is discovered to be able to self-organize to generate a lateral toroid moment. Interestingly, this behavior has never been reported for ferromagnetic vortices (to the best of our knowledge). We have also performed calculations for pure $\mathrm{PbTiO}_{3}$ nanodisks and found that the $\mathrm{G}_{y}$ toroid moment robustly occurs, revealing that the existence of phase II is not related to the composition fluctuation in PZT. Assuming that dipoles respond by rotating collectively toward the field direction, one would anticipate that the drastic decrease of the $\mathrm{G}_{z}$ moment shall be accompanied by a sharp rise in the polarization. Surprisingly, the net $\mathrm{P}_{z}$ polarization in Fig 19 apparently does not feel the drastic variation of the $\mathrm{G}_{z}$, 
and remains, to the naked eye, fairly smooth. As the electric field exceeds a second critical value $\mathrm{E}_{c, 2}=2.8 \mathrm{~V} / \mathrm{nm}$, all $\mathbf{G}$ components vanish and the system becomes a phase of pure polarization. Low-symmetry phase II thus acts as the key intermediate state for bridging the transformation from phase I (of the same cylindrical symmetry as the initial vortex) to the destination phase of uniform polarization (that also has cylindrical symmetry).

The evolution of toroid moment during the vortex transformation is also reflected in dielectric $\chi_{33}$ susceptibility (Fig.1b), showing a noticeable hump at $\mathrm{E}_{c, 1}$ field. Furthermore, near the critical $\mathrm{E}_{c, 2}$ field where vortex collapses, the $\chi_{33}$ coefficient exhibits a sharp decline, demonstrating that dipoles in vortex phase generate a notably larger dielectric response than dipoles in ferroelectric phase do.

We now present microscopic understanding of the puzzling collective behaviors that FE vortex displays in Fig, 17 along the transformation path. Fig,2 shows snapshots of the dipole configurations corresponding to four selected fields $\mathrm{E}_{i}(\mathrm{i}=1 \sim 4)$ labelled in Fig [1]. Comparison of the dipole patterns at $\mathrm{E}_{1}$ and $\mathrm{E}_{2}$ fields shows that the vortex response in phase I is characterized by collective rotation of dipoles towards the field direction. Note that at $\mathrm{E}_{2}$ field the vortex pattern on the $x y$ plane maintains a cylindrical symmetry. The rotation is thus symmetry conforming in the sense that dipoles within the same distance from the central axis respond equivalently. Another important conclusion from simulations concerns where the polarization nucleates, since the phase transition involve two different order parameters. We find that the $z$-axis polarization is nucleated at the center of the $\mathrm{FE}$ vortex. In other words, formation of polarization begins with and is initiated by the rotation of those dipoles near the cylindrical axis, which is consistent with the explanation that a large strain exists in the vortex center and the rotation reduces this strain energy.

As the field changes slightly from $E_{2}$ to $E_{3}$, two striking phenomena occur: (1) The dipole components in the $x y$ plane cease to exhibit cylindrical symmetry (see the $\mathrm{E}_{3}$ pattern, lower panel, Fig.21). In fact, the inplane components disappear remarkably for dipoles within a certain, but not across the entire $360^{\circ}$ degree of, azimuthal angle. (2) A lateral $\mathrm{G}_{y}$ vortex for which the corresponding toroid moment points at the $y$-axis - starts to nucleate near the right-side surface on the $x z$ plane (see the $\mathrm{E}_{3}$ pattern, upper panel of Fig,2). Note that this $\mathrm{G}_{y}$ vortex forms neither at the cylindrical axis, nor at the same side as the azimuthal angle where inplane dipole components are annihilated, in order to avoid the large strain energy when vortex develops. 
Further increase of the electric field from $\mathrm{E}_{3}$ to $\mathrm{E}_{4}$ leads to the widening of the annihilation angle (the $\mathrm{E}_{4}$ pattern, lower panel, Fig.2) and full development of the lateral $\mathrm{G}_{y}$ vortex. The system at the $\mathrm{E}_{4}$ field is thus characterized by formation of a vortex-free ferroelectric region on one side - and a vortex region with perpendicular $\mathrm{G}_{y}$ moment on the other side (the $\mathrm{E}_{4}$ pattern, upper panel, Fig,2). Interestingly, despite that formation of the lateral $\mathrm{G}_{y}$ vortex forces some dipoles to point opposite to the field direction between $\mathrm{E}_{3}$ and $\mathrm{E}_{4}$ fields, the net $\mathrm{P}_{z}$ polarization nonetheless remains increasing as seen in Fig.1 $1 \mathrm{a}$, consistent with the Le Chatelier's principle[7]. Finally, when electric field continues to increase above $\mathrm{E}_{4}$, the ferroelectric region expands by extruding the center of the $\mathrm{G}_{y}$ vortex toward the right-side surface, and this $\mathrm{G}_{y}$ vortex eventually disappears at critical $\mathrm{E}_{c, 2}$ field.

Though complex, the dipole behaviors in Fig, 2 have a simple explanation, namely they result from the competition between the applied electric field and depolarizing field. For phase II at $E_{c, 1}<E<E_{c, 2}$, these two fields becomes comparable, and the nanodisk desires to reduce the $z$-axis depolarizing field by forming lateral $\mathrm{G}_{y}$ vortex. Indeed, as shown in Fig 2 for the system at $\mathrm{E}_{4}$ field, the depolarizing field in phase II is mainly confined to the ferroelectric region while being reduced in the vortex region. Meanwhile, to enhance the interaction between polarization and the external field, those dipoles in the ferroelectric region favor to rotate toward the $z$-axis more than the dipoles in the $\mathrm{G}_{y}$ vortex region, leading to the collective phenomenon that the inplane $\mathrm{G}_{z}$ vortex is annihilated without maintaining the azimuthal symmetry.

The presence of phase II (with toroid moment rotated by $90^{\circ}$ with respect to the initial $\mathrm{G}_{z}$ vortex) raises a question of what may happen when one starts with this phase (e.g., at $\mathrm{E}=2 \mathrm{~V} / \mathrm{nm}$ in Fig, (1a) and then decreases the external field. Our simulations reveal that, regardless of whether the field is gradually reduced or suddenly switched off, the system in phase II does not transform back to the initial state of $\mathrm{G}_{z}$ moment, and instead is trapped in the $\mathrm{G}_{y}$ vortex state. More specifically, as the field is reduced, the $\mathrm{G}_{y}$ vortex at the $\mathrm{E}_{4}$ field in Fig 2 grows by moving its core toward the cylindrical axis (not toward the right-side surface), resulting in a pure vortex state with a $G_{y}$ moment and $\mathbf{P}=0$. This leads to a hysteresis as shown in the left plot of Fig.1k, which is interesting in the sense that (i) the hysteresis is caused by toroid moment, not polarization; (ii) it exists in a single particle of nanometer size; (iii) during the hysteresis the toroid moment is rotated rather than switched to the opposite. We further find that the $\mathrm{G}_{y}$ and $\mathrm{G}_{z}$ phases at zero field in Fig.1p are very 
close in energy; the latter is lower by $\sim 1 \mathrm{meV}$ per 5 -atom unit cell. Trapping of the system in the $\mathrm{G}_{y}$ state also suggests that this state is stable and surrounded by energy barrier. To confirm this, the system of the $\mathrm{G}_{y}$ phase is heated at zero field to a chosen temperature $\tilde{T}$ and then cooled down to $64 \mathrm{~K}$. We found that only when $\tilde{T}$ is above $500 \mathrm{~K}$ the $\mathrm{G}_{y}$ phase is able to overcome the barrier and becomes the $\mathrm{G}_{z}$ phase (see the right plot of Fig.1k).

Our analysis shows that, in addition to the reduction of the depolarizing field, there is another factor that facilitates the transformation from phase I into phase II, that is, the interaction between local mode and strain. Fig.11 depicts the strain components at different fields. At zero field the lattice of the vortex state is pseudo-tetragonal with $c / a$ ratio less than 1, i.e., with strain components $\eta_{x x}=\eta_{y y}>\eta_{z z}$, because all dipoles are lying in the $x y$ plane. As the field increases, the $c / a$ rises as a result of the polarization-strain coupling. Notably, the field at which $c / a$ becomes 1 (i.e., the system becomes pseudocubic) is very close to the critical $\mathrm{E}_{c, 1}$ field where phase I is transformed into phase II. The mode-strain coupling[19] $-\sum_{i}|B|\left(\eta_{x x} u_{i x}^{2}+\eta_{y y} u_{i y}^{2}+\eta_{z z} u_{i z}^{2}\right)$ (where B is the coupling strength) advances the transition into phase II largely due to the increase in atomic volume, $\Delta \Omega / \Omega_{0}=\eta_{x x}+\eta_{y y}+\eta_{z z}$, which is nearly a constant in phase $\mathrm{I}$ and rises sharply for $\mathrm{E}>\mathrm{E}_{c, 1}$ (Fig!1d).

We next address how the diameter of nanodisk may influence the vortex transformation. Interestingly, we find that there is a critical size $\mathrm{d}_{c}=17$, below which the transformation path turns out to be different. Simulation results of a $\mathrm{d}=9$ disk (not shown here) reveal that the vortex undergoes a continuous transformation into a single domain ferroelectric phase, without the bridging phase II. The dielectric $\chi_{33}$ coefficient for this disk at zero field is determined to be 55 (Fig,1 1 ) and is considerably larger than the value of 25 in the $\mathrm{d}=19$ disk, showing that the same electric field is able to induce a much larger polarization in smaller disks. The critical $\mathrm{E}_{c, 2}$ field in the $\mathrm{d}=9$ disk decreases to $1.3 \mathrm{~V} / \mathrm{nm}$. One main difference between $\mathrm{d}=9$ and $\mathrm{d}=19$ nanodisks is the depolarizing effect which is small in the former and thus allows a symmetry conforming transformation.

Finally we point out that the predicted FE-vortex responses have important technological implication. The quadratic law, $G_{z}(E)=G_{0}-\sigma E^{2}$, tells us that, when a $\mathrm{FE}$ nanodisk is exposed to an alternating field $\mathbf{E}(t)=\mathbf{E}_{0} \cos \omega t$, the toroid moment $\mathbf{G}(t)$ will respond with a double frequency $2 \omega$, and its radiation field can be separated from the field of the vibrating polarization which responds only with $\omega$. The signal with $2 \omega$ frequency may further tell us whether it is associated with moment $\mathbf{G}$ or $-\mathbf{G}$, since the latter field is phase shifted by 
$\pi$.[22] This may thus open a novel approach by using electromagnetic fields of pulse lasers to probe and/or read FE vortex state. Compared to mechanical approach using piezoelectric force microscope tips, the optic approach is fast and can be performed simultaneously in a large quantity.

In summary, (i) the transformation between vortex and ferroelectric phases in FE particles is predicted to follow an unusual and symmetry non-conforming path, which is a macroscopic manifestation of the delicate competition of microscopic interactions. A new structure phase, with rotated vortex moment and coexisting polarization, was revealed as the intermediate phase bridging the transformation. (ii) We discovered a striking collective phenomenon leading to the annihilation of FE vortex, namely that the vortex does not disappear shellby-shell, but in a peculiar azimuthal annihilation mode. (iii) The center of FE vortex plays a crucial role for the nucleation of polarization. (iv) The existence of an interesting hysteresis, caused by toroid moment, may act as a previously unknown channel for energy dissipation and dielectric loss. (v) Our simulations further suggest an innovative optic approach for reading/probing toroid moment.

This work was supported by the National Science Foundation and Office of Naval Research. The computing facilities were provided by the Center for Piezoelectrics by Design.

[1] T. Shinjo, T. Okuno, R. Hassdorf, K. Shigeto, T. Ono, Science 289, 930 (2000).

[2] A.A. Abrikosov, Sov. Phys. JETP 9, 1174 (1957).

[3] K.W. Madison, F. Chevy, W. Wohlleben, J. Dulibard,, Phys. Rev. Lett. 84, 806 (2000).

[4] V.L. Ginzburg, A.A. Gorbatsevich, Yu.V. Kopayev, and B.A. Volkov, Solid State Commun. 50, 339 (1984).

[5] I.I. Naumov, L. Bellaiche, and H. Fu, Nature 432, 737 (2004).

[6] D.D. Fong, G.B. Stephenson, S.K Streiffer, J.A. Eastman, O. Auciello, P.H. Fuoss, and C. Thompson, Science 304, 1650 (2004).

[7] L.D. Landau and E.M. Lifshitz, Statistical Physics, Part 1, (Pergamon, New York, 1980).

[8] R.E. Cohen, Nature 358, 136 (1992).

[9] J.F. Scott, Ferroelectric Memories (Springer, Berlin, 2000).

[10] M.E. Lines and A.M. Glass, Principles and Applications of Ferroelectrics and Related Materials 
(Clarendon, Oxford, 1979).

[11] K. Uchino, Pizoelectric Actuators and Ultrasonic Motors (Kluwer Academic, Boston, 1996).

[12] S. O’Brien, L. Brus, and C.B. Murray, J. Am. Chem. Soc. 123, 12085 (2001).

[13] W.S. Yun, J.J. Urban, Q. Gu, and H. Park, Nano Lett. 2, 447 (2001).

[14] F.D. Morrison et al., Rev. Adv. Mater. Sci. 4, 114 (2003).

[15] F. Jona and G. Shirane, Ferroelecric Crystals (Dover Pub., New York, 1993), p 374.

[16] E. Yu. Vedmedenko, A. Ghazali, and J.-C. S. Levy, Phys. Rev. B 59, 3329 (1999).

[17] R.P. Cowburn et al., Phys. Rev. Lett. 83, 1042(1999).

[18] H. Fu and L. Bellaiche, Phys. Rev. Lett. 91, 257601 (2003).

[19] W. Zhong, D. Vanderbilt, and K. M. Rabe, Phys. Rev. Lett. 73, 1861 (1994).

[20] L. Bellaiche, A. Garcia, and D. Vanderbilt, Phys. Rev. Lett. 84, 5427 (2000).

[21] A. Garcia and D. Vanderbilt, Appl. Phys. Lett. 72, 2981 (1998).

[22] V.M. Dubovik, M.A. Martsenyk, N.M. Martsenyk, J. Magn. Magn. Mater. 145, 211 (1995). 

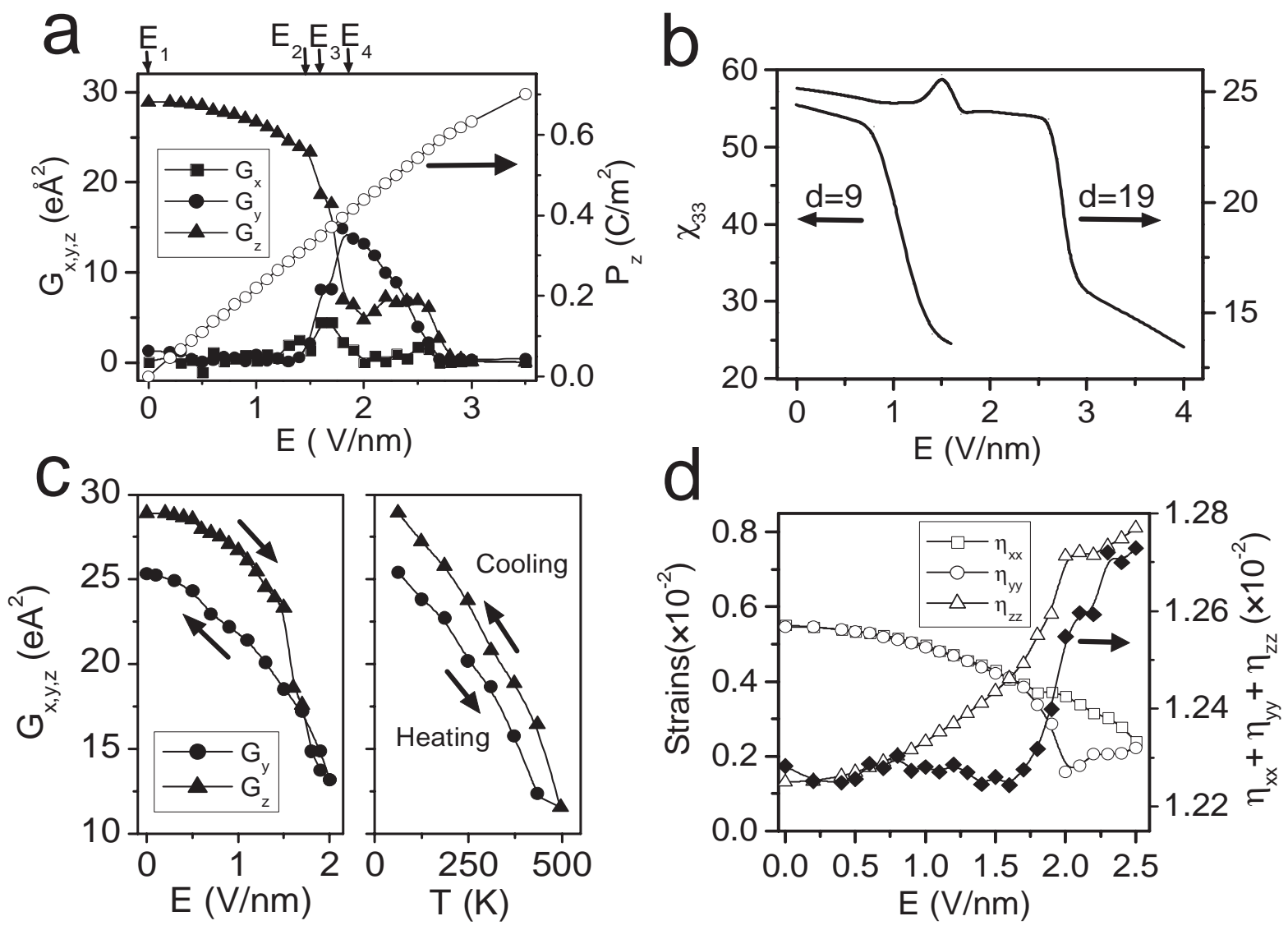

FIG. 1: (a) Toroid moment $\mathbf{G}$ (using the left vertical axis) and net polarization $\mathrm{P}_{z}$ (using the right vertical axis) in a $\mathrm{d}=19$ disk. Arrows on the top horizontal axis indicate the selected electric fields for which dipole configurations are analyzed. (b) Dielectric $\chi_{33}$ susceptibility in the $d=9$ disk (using the left vertical axis) and in the $\mathrm{d}=19$ disk (using the right vertical axis). (c) Left: hysteresis of toroid moment caused by increasing and then decreasing the electric field in $\mathrm{d}=19$ disk; Right: transformation of the $\mathrm{G}_{y}$ vortex into a $\mathrm{G}_{z}$ vortex by heating the system to $500 \mathrm{~K}$ and then cooling down. (d) Strain components $\eta_{x x}, \eta_{y y}$ and $\eta_{z z}$ (using the left vertical axis) and volume expansion $\eta_{x x}+\eta_{y y}+\eta_{z z}$ (filled symbols, using the right vertical axis) in the $\mathrm{d}=19$ disk. The horizontal axis in (a), (b), and (d) is the strength of electric field. 

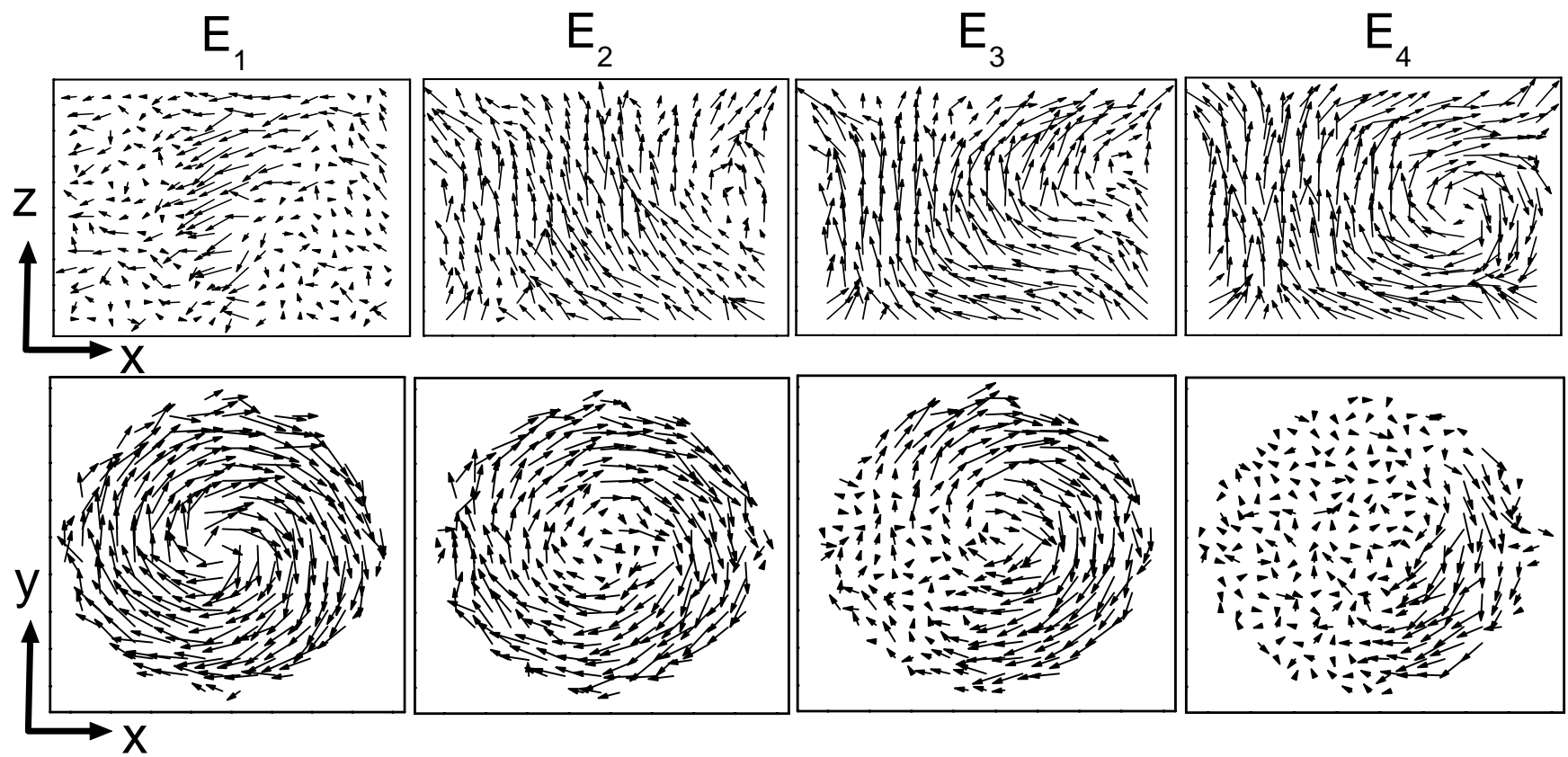

FIG. 2: Configurations of local dipoles on the central $x y$ cross section (lower panel) and on the central $x z$ cross section (upper panel) in the $\mathrm{d}=19$ disk, at selected fields $\mathrm{E}_{1}, \mathrm{E}_{2}, \mathrm{E}_{3}$ and $\mathrm{E}_{4}$ as marked by the arrows in Fig[1a. The magnitude of each dipole is enlarged for clarity. 